\title{
Virtual colonoscopy performed poorly in detecting colorectal neoplasia
}

Cotton PB, Durkalski VL, Pineau BC, et al. Computed tomographic colonography (virtual colonoscopy): a multicenter comparison with standard colonoscopy for detection of colorectal neoplasia. JAMA 2004;291:1713-9.

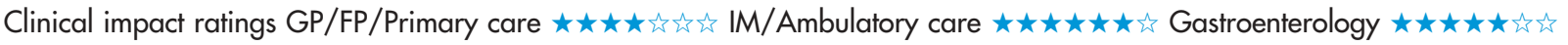

In patients presenting for colonoscopy, what is the accuracy of computed tomographic (CT) colonoscopy (virtual colonoscopy [VC]) in detecting colororectal neoplasia?

\section{METHODS}

Design: blinded, non-inferiority comparison of VC with
conventional colonoscopy.
Petting: 8 clinical centres in the US and 1 centre in the UK.
for colonoscopy because of overt and occult rectal bleeding,
change in stool habit, abdominal pain, or surveillance after
polypectomy. Patients who had had colonoscopy within the past
3 years were excluded.
Description of tests: the colon was insufflated with room air or
carbon dioxide. VC was done using 2 and 4 section CT scanners
with nominal slice thicknesses of 2.5 or 5 mm and reconstruction
increments of 1.5 or 1 mm, depending on equipment. Scans were
read in 2 dimensional slices and 3 dimensional snapshot
reconstructions when necessary. Radiologist interpretations were
recorded in a sealed envelope for each colon segment.
Conventional colonoscopy was done within 2 hours of VC.
Endoscopists were blinded to VC results during insertion of the
colonoscope. After each segment was examined and results
recorded, the VC results for that segment were revealed, allowing
the endoscopist to reexamine any discrepancy.
Diagnostic standard: initial VC results, additional findings on
conventional colonoscopy after segmental unblinding to the VC
results, and results of additional diagnostic tests done later when
clinically indicated.
Outcomes: sensitivity and specificity of VC and conventional
colonoscopy in detecting lesions $\geqslant 6$ mm.

For correspondence: Dr P B Cotton, Medical University of South Carolina, Charleston, SC, USA. cottonp@musc.edu

Source of funding: Office of Naval Research, US Department of Defense.

\section{MAIN RESULTS}

827 lesions were detected in 308 patients. The prevalence of lesions 1-5 $\mathrm{mm}, 6-9 \mathrm{~mm}$, and $\geqslant 10 \mathrm{~mm}$ was $79 \%, 14 \%$, and $6.5 \%$, respectively. The sensitivity of VC for detecting lesions of any size was much less than that of conventional colonoscopy (table).

\section{CONCLUSION}

In patients presenting for colonoscopy, virtual colonoscopy was inferior to conventional colonoscopy in detecting colorectal neoplasia.

Abstract and commentary also appear in ACP Journal Club

\section{Commentory}

$s$ it time for VC to be included among the screening options? If I had had only the Pickhardt study to guide me, I might have been tempted. But the Cotton study reminds us that the test is not yet ready for general use. Sensitivity and specificity in ordinary circumstances are not high enough. Also, cost and the consequences to patients with abnormal results have not yet been vigorously examined. Abnormal VC results must be followed up with another procedure (conventional colonoscopy), with its own demanding preparation and costs. As for the strength of the evidence of effectiveness, there are no studies of whether screening VC prevents colorectal cancer deaths. However, the medical community seems willing to accept that polyp detection by any means, followed by removal, leads to fewer cases colorectal cancer - by generalising from studies in which both polyp or cancer detection rates and colorectal cancer deaths have been reported.

VC is already available in some centres and marketed to the general public. But it is not yet included in guidelines. As the technology continues to improve and if more studies of recent generation technology are as persuasive as the Pickhardt study, it may be just a matter of time before VC is added to the list of accepted screening options. The Pickhardt study suggests that the time might not be far away, and the Cotton study reminds us that the time has not yet arrived.

Continued on next page.

Test characteristics of virtual colonoscopy (VC) and conventional colonoscopy (CC) in detecting colorectal neoplasia*

\begin{tabular}{llllll}
\hline Test & Lesion size & Sensitivity (95\% Cl) & Specificity (CI) & +LR & -LR \\
\hline VC & $1-5 \mathrm{~mm}$ & $14 \%(10$ to 18$)$ & $91 \%(87$ to 94$)$ & 1.42 & 0.96 \\
& $\geqslant 6 \mathrm{~mm}$ & $39 \%(10$ to 48$)$ & $91 \%(88$ to 93$)$ & 4.11 & 0.67 \\
& $6-9 \mathrm{~mm}$ & $30 \%(20$ to 40$)$ & $93 \%(91$ to 95$)$ & 4.35 & 0.75 \\
$\mathrm{CC}$ & $\geqslant 10 \mathrm{~mm}$ & $55 \%(40$ to 70$)$ & $96 \%(94$ to 98$)$ & 13.75 & 0.47 \\
& $1-5 \mathrm{~mm}$ & $97 \%(95$ to 99$)$ & $100 \%$ & $\infty$ & 0.03 \\
& $\geqslant 6 \mathrm{~mm}$ & $99 \%(97$ to $>99.9)$ & $100 \%$ & $\infty$ & 0.01 \\
& $6-9 \mathrm{~mm}$ & $99 \%(96$ to $>99.9)$ & $100 \%$ & $\infty$ & 0.01 \\
& $\geqslant 10 \mathrm{~mm}$ & $100 \%$ & $100 \%$ & $\infty .0$ \\
\hline
\end{tabular}

*Diagnostic terms defined in glossary; LRs calculated from data in article. 